\title{
Quantifying the fraction of alanine residues in an $\alpha$-helical conformation in hornet silk using solid-state NMR
}

\author{
Tsunenori Kameda
}

Here, we demonstrate the first successful isotope labeling of Ala carbons in hornet silk produced by the larvae of Vespa (Vespinae, Vespidae) mandarinia. This labeled hornet silk was examined by high-resolution ${ }^{13} \mathrm{C}$ solid-state NMR, and it was found that the fraction of Ala residues in $\alpha$-helical conformations compared with Ala residues in the overall conformation of hornet silk can be quantitatively determined from Ala $C_{\alpha}$ NMR peaks. The value for this $\alpha$-helical Ala fraction is close to that of the fraction of Ala residues in coiled-coil structures estimated in the four major hornet silk proteins by coiled-coil prediction analysis. This result indicates that most of the Ala residues in $\alpha$-helices occur in those $\alpha$-helices with a coiled-coil structure, and that the number of Ala residues in $\alpha$-helices without a coiled-coil structure is small. Moreover, coiled-coil prediction analysis indicated that the potential coiled-coil domains are located only in the central portion of the protein chains of the major hornet silk proteins. From these results, we confirmed that the $\alpha$-helical conformation mostly forms in the central portion of the hornet silk chains, whereas the ends of the protein chains are nearly devoid of $\alpha$-helical structure. We deduce that the ends of the protein chains would preferentially adopt a $\beta$-sheet conformation.

Polymer Journal (2012) 44, 876-881; doi:10.1038/pj.2012.93; published online 23 May 2012

Keywords: conformation; hornet (Vespa) silk; isotope labeling; solid-state NMR

\section{INTRODUCTION}

It is common knowledge that silkworms and spiders produce silk. ${ }^{1}$ However, it is not widely known that the larvae of hornets (Vespa, Vespinae, Vespidae) also spin silk (hornet silk) and produce cocoons before pupation. ${ }^{2-4}$ The structure of hornet silk has been reported in our recent studies. ${ }^{5-12}$ The amino-acid sequences of six types of hornet silk proteins of Vespa simillima xanthoptera Cameron have been determined. ${ }^{5,6,10}$ Of these six proteins, four are major and have similar characteristics. These four major proteins consist of an alanine(Ala)-rich domain at the center of the chain and serine(Ser)rich domains at both ends of the chain. ${ }^{6,10}$

Hornet silk proteins contain both $\alpha$-helices and $\beta$-sheets. ${ }^{12}$ In addition, hornet silk contains coiled-coil structures, ${ }^{10}$ which are a common feature of the silks of the social Hymenoptera (bees, ants and hornets)., ${ }^{3,13}$ The coiled-coil structure consists of several $\alpha$-helices wound around each other. ${ }^{14}$ The $\alpha$-helices that form the coiled-coils are characterized by a 7-residue (heptad) repeat, conventionally denoted as $(a b c d e f g)_{n}$. The positions $a$ and $d$ are normally occupied by hydrophobic residues. ${ }^{15}$ Using coiled-coil prediction programs to search the heptad repeats of the primary structures, the location of potential coiled-coil domains in hornet silk proteins was predicted. ${ }^{10}$ The results indicated that coiled-coil structures exist only in the Alarich domain, and not in the Ser-rich domains. From this result, we confirmed that $\alpha$-helices exist in the Ala-rich domain, as the $\alpha$-helix is a prerequisite for the formation of a coiled-coil structure. Indeed, Ala is known to be an amino acid that stabilizes $\alpha$-helices. ${ }^{16}$ We believe that the Ala-rich domain adopts an $\alpha$-helical conformation, whereas Ser tends to be $\beta$-sheet stabilizing. ${ }^{8}$ However, to date, we have yet to adequately demonstrate the absence of $\alpha$-helical conformation in the Ser-rich domain. Even if no coiled-coils exist in the Ser-rich domain, the existence of $\alpha$-helical conformations without a coiled-coil structure in the Ser-rich domains cannot be discounted.

As Ala is the most abundant amino acid in hornet silk and is dispersed throughout the entire protein, Ala residues are present not only in the Ala-rich domain but also the Ser-rich domains. ${ }^{10}$ Therefore, information on the entire structure of hornet silk proteins, including the Ser-rich domains, is expected to be obtained from a structural analysis of the Ala residues in hornet silk. We believed that the existence of $\alpha$-helical conformations without a coiled-coil structure would be clarified from a comparison of the two types of Ala fractions in the total Ala in hornet silk: the fraction of Ala residues within $\alpha$-helical conformations and the fraction of Ala residues within coiled-coil structures. If the fraction of Ala residues within $\alpha$-helical conformations in hornet silk is greater than that in coiled-coil structures, then $\alpha$-helical conformations without a coiledcoil structure exist in hornet silk. In this case, there is a probability 
that $\alpha$-helical conformations without a coiled-coil structure exist in the Ser-rich domains. If, however, the Ala fraction within $\alpha$-helical conformations is equal to that in coiled-coil structures, then it can be assumed that in hornet silk there is only a small amount of $\alpha$-helical conformation without a coiled-coil structure. Accordingly, the probability of $\alpha$-helical conformations without coiled-coil structures existing in the Ser-rich domains is small.

To experimentally determine the fraction of the total Ala residues in hornet silk that are in an $\alpha$-helical conformation, high-resolution ${ }^{13} \mathrm{C}$ solid-state NMR is an ideal tool, as all the peaks attributable to Ala $\mathrm{C}_{\alpha}, \mathrm{C}_{\beta}$ and carbonyl carbon $(\mathrm{C}=\mathrm{O})$ in hornet silk cocoon can be discriminated in a high-resolution ${ }^{13} \mathrm{C}$ solid-state $\mathrm{NMR}$ spectrum. ${ }^{12}$ In addition, the differences in the NMR chemical shift values of Ala carbons in the $\alpha$-helical conformation and other conformations are large enough to separate into each peak. ${ }^{6}$ Each of the Ala $C_{\alpha}, C_{\beta}$ and $\mathrm{C}=\mathrm{O}$ peaks in the high-resolution solid-state NMR spectrum of hornet silk gives identical structural information on the Ala residues; that is, each of the three peaks consists of two components: $\alpha$-helical and $\beta$-sheet conformations. In addition, all of the three peaks indicate that the amount of Ala in $\alpha$-helices is greater than that in $\beta$-sheets; that is, each Ala carbon in the $\alpha$-helical conformation resonates more strongly than those in $\beta$-sheets. However, the ratios of the peak intensities of Ala in $\alpha$-helices and $\beta$-sheets differ among Ala $C_{\alpha}, C_{\beta}$ and $\mathrm{C}=\mathrm{O}$. Accordingly, the quantitative ratio between Ala residues in $\alpha$-helices and $\beta$-sheets has been difficult to determine from intensities of the peaks. This difference may be because Ala peaks overlap with the other peaks. When the peak overlaps with other peaks, the observed peak intensity becomes stronger than the genuine peak intensity. Quantitative analysis of the abundance ratio for $\alpha$-helical $\mathrm{Ala}$ and $\beta$-sheet Ala is necessary to experimentally determine the fraction of the total Ala residues within the $\alpha$-helical conformation in hornet silk.

To avoid the issues of peak overlap in NMR spectra, selective isotope labeling is often effective. Among the numerous peaks in the NMR spectrum of silk, only the peaks attributable to isotope labeled amino acids are enlarged. Isotope labeling of amino acids in silk fibroins from moth larvae and spiders has been well studied. ${ }^{17-21}$ One method to biosynthetically label silkworm silk is through the use of an artificial diet supplemented with isotope-labeled amino acids during the fifth-instar larval stage. Isotope-labeled silk fibroin can then be collected from the cocoon. ${ }^{19}$ Using this method to label hornet larvae, isotope-labeled hornet cocoons are expected to be obtained.

In this study, we attempted to obtain isotope-labeled hornet silk in which only the Ala carbons were substituted by carbon-13 and to quantitatively analyze the fraction of Ala residues in hornet silk in an $\alpha$-helical conformation by means of solid-state NMR. Further, in order to clarify the existence of $\alpha$-helical conformations without a coiled-coil structure, this fraction was compared with the fraction of Ala residues in hornet silk within coiled-coil structures estimated using data calculated using a coiled-coil prediction program. Finally, the molecular structure of the Ala-rich and Ser-rich domains in hornet silk is discussed based on the results obtained.

\section{EXPERIMENTAL PROCEDURE}

\section{Silk samples}

Nests of V. mandarinia and V. simillima xanthoptera Cameron were collected from the field in the city of Tsukuba, Japan during 2011 in the autumn, which is the active season for this hornet. The hornet silk was carefully gathered from the comb cells.

The fifth-instar $V$. mandarinia larvae in the collected nests were bred until they started spinning silk. The larvae were successfully bred on a diet of larval saliva, which consists mainly of amino acids ${ }^{22}$ and is suitable as bait. It is widely known that adult hornets subsist on a diet of larval saliva, as they are unable to consume solid foods because of their constricted abdomens. ${ }^{23}$

Figure 1 shows a schematic representation of the process by which hornet cocoons containing $\left[{ }^{13} \mathrm{C}_{3}\right]$ Ala-labeled silk proteins are prepared using isotopelabeled amino acid. At first, the target larva used to administer the isotopically labeled amino acid was selected from mature larva (fifth instar), which was called the 'labeling larva' (Figure 1b). In addition, larval saliva was also collected from hornet larvae other than the labeling larvae (Figure 1a). $D L$-alanine $(10 \mathrm{mg})$ with uniformly labeled ${ }^{13} \mathrm{C}\left(\left[{ }^{13} \mathrm{C}_{3}\right] \mathrm{Ala}\right)$ purchased from Santa Cruz Biotechnology, Inc. (Santa Cruz, CA, USA) was dissolved in $125 \mu \mathrm{l}$ of larval saliva. The $125 \mu \mathrm{l}$ of $\left[{ }^{13} \mathrm{C}_{3}\right] \mathrm{Ala}$ saliva solution was orally administered to the labeling larvae (Figure 1) three times per day, and this procedure was continued until the fifth-instar larvae started spinning silk (7-10 days). $\left[{ }^{13} \mathrm{C}_{3}\right]$ Ala-free saliva solution was also frequently administered to the labeling larvae.

\section{Solid-state NMR measurements}

High-resolution solid-state ${ }^{13} \mathrm{C}$ NMR spectra were recorded using an Avance 600 WB (Bruker, Karlsruhe, Germany), with a magnetic field of 14.1T. The spectrometers were operated at a ${ }^{13} \mathrm{C}$ NMR frequency of $150.94 \mathrm{MHz}$. The samples were placed in a solid-state probe and spun at a magic-angle spinning (MAS) frequency of $10.0 \mathrm{kHz}$ in a $4.0-\mathrm{mm}-\phi$ zirconia rotor. For the ${ }^{13} \mathrm{C} 90^{\circ}$ single pulse (direct polarization (DP)) experiments, the $90^{\circ}$ pulse length for the ${ }^{13} \mathrm{C}$ measurements was $3.75 \mu \mathrm{s}$. In the cross-polarization (CP) experiments, a ${ }^{1} \mathrm{H} 90{ }^{\circ}$ pulse length of $3.5 \mu$ s and ${ }^{1} \mathrm{H}-{ }^{13} \mathrm{C} \mathrm{CP}$ contact of $70 \mathrm{kHz}$ was employed. The high-power ${ }^{1} \mathrm{H}$ decoupling using the SPINAL-64 method was employed for both DP and CP methods. The repetition time for the CP experiments was set at $3.0 \mathrm{~s}$. All the spectra were calibrated using adamantane as the standard, and the chemical shift of the adamantane $\mathrm{CH}_{2}$ peak appearing at 29.5 p.p.m. was referenced to the tetramethylsilane peak appearing at 0 p.p.m.

\section{Bioinformatics analysis}

The Ala and Ser-rich domains in the amino-acid sequences of hornet silk proteins were determined by the PROSITE database of protein domains (http://kr.expasy.org/prosite). The coiled-coil formations in the amino-acid sequences of the hornet silk proteins were predicted using the Multicoil program (http://multicoil.lcs.mit.edu/cgi-bin/multicoil) and MARCOIL (http://toolkit.tuebingen.mpg.de/marcoil)

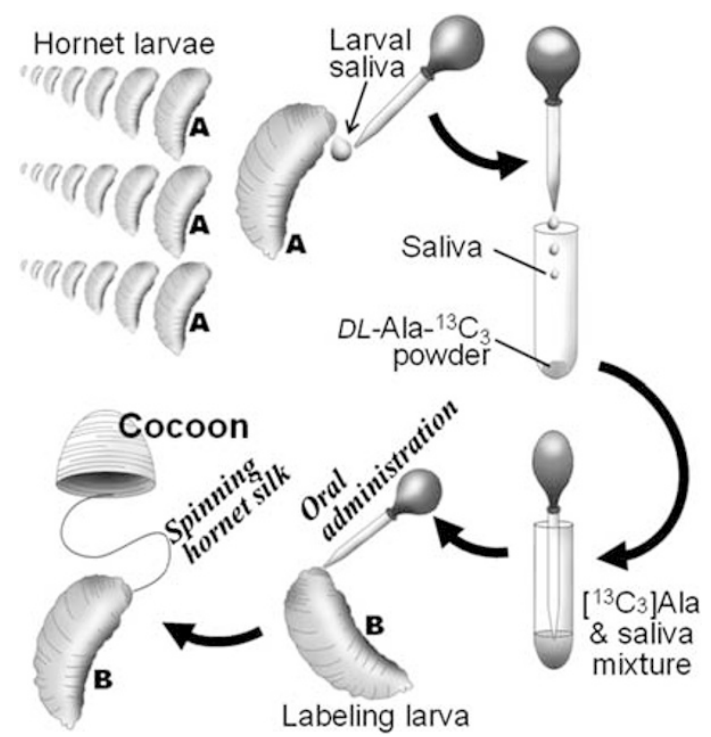

Figure 1 Schematic representation of the preparation of hornet cocoons containing $\left[{ }^{13} \mathrm{C}_{3}\right]$ Ala-labeled silk proteins. $A$ and $B$ indicate the larvae used for saliva collection and the larva used for labeling, respectively. 


\section{RESULTS AND DISCUSSION}

\section{Isotope labeling of hornet silk}

The ${ }^{13} \mathrm{C}$ CP-MAS spectra of hornet cocoons produced by larvae that were orally administrated $D L-\mathrm{Ala}^{13} \mathrm{C}_{3}$ are shown in Figure $2 \mathrm{~b}$ together with that of native cocoons (natural abundance) produced by the larvae of $V$. mandarinia (Figure 2a). An apparent difference was observed in these spectra, and some specific peaks were only enhanced in Figure 2b. As the positions of these enhanced peaks were in an area similar to those peaks in the ${ }^{13} \mathrm{C} C \mathrm{CP}-\mathrm{MAS}$ NMR spectrum of $D L$-Ala- ${ }^{13} \mathrm{C}_{3}$ (Figure 2c), the enhanced peaks in Figure $2 \mathrm{~b}$ were believed to be attributable to Ala carbons. However, there is a possibility that these enhanced peaks were merely attributable to the administered $D L$-Ala that adhered to the cocoons. However, this possibility was refuted by the peak widths and chemical shift values of the enhanced peaks (Figure 2b), especially the chemical shifts of Ala $\mathrm{C}_{\alpha}$ and $\mathrm{C}_{\beta}$, which were different from the peaks of $D L$-Ala (Figure 2c). Moreover, when the structure of hornet silk was changed from $\alpha$-helix to $\beta$-sheet by

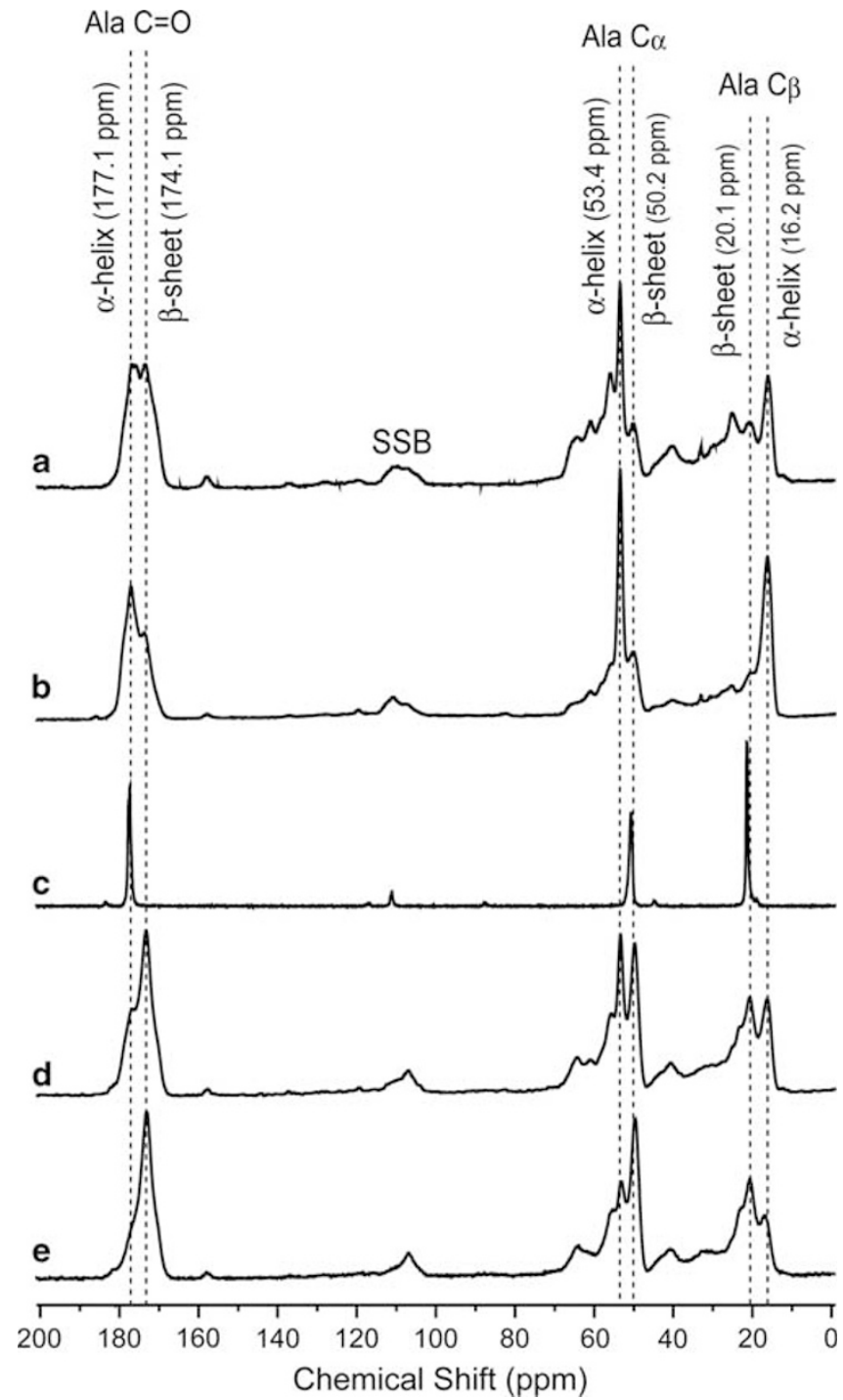

Figure 2 The ${ }^{13} \mathrm{C}$ CP-MAS NMR spectra of cocoons produced by hornet larvae (a) and $D L$-Ala- ${ }^{13} \mathrm{C}_{3}$ administered hornet larvae (b) of $V$. mandarinia, together with the spectra of $D L-A l a-{ }^{13} C_{3}$ powder (c) and the cocoon in Figure $2 \mathrm{~B}$ after treatment in hot water at $85^{\circ} \mathrm{C}$ for $8 \mathrm{~h}(\mathrm{~d})$ and in steam at $121^{\circ} \mathrm{C}$ for $20 \mathrm{~min}$ (e). SSB, spinning side band. incubation in hot water, ${ }^{6}$ all of the enhanced peaks were also shifted (Figure $2 \mathrm{~d}$ and e). The chemical shifts of the enhanced peaks before and after structural changes are in agreement with those of Ala carbons in the hornet silk within $\alpha$-helix and $\beta$-sheet, respectively. ${ }^{6}$ From these results, we confirmed that the enhanced peaks in Figure $2 \mathrm{~b}$ were not attributable to the administrated $D L$-Ala, but were attributable to the $\mathrm{Ala} \mathrm{C}_{\alpha}, \mathrm{C}_{\beta}$ and $\mathrm{C}=\mathrm{O}$ incorporated in hornet silk, that is, the orally administered $\left[{ }^{13} \mathrm{C}_{3}\right] \mathrm{Ala}$ was biosynthetically incorporated into the hornet silk proteins.

It is widely known that Ala is metabolized in the production of other amino acids. ${ }^{24}$ In spiders, Ala is metabolized in the production of other amino acids during the biosynthesis of silk proteins, and the carbons labeled in Ala are scrambled into other amino acids in the silk proteins. ${ }^{20,21}$ Conversely, in hornet silk, the Ala carbons are welllabeled, whereas other carbons are less labeled, as shown in Figure 2b. This suggests that the most of the Ala is fixed in hornet silk without further metabolism. Even though the amount is small, there is a possibility that some of the Ala is metabolized; however, the product (pyruvic acid) may not be used for the premetabolic compound of amino acids incorporated into hornet silk proteins. A possible reason for the low Ala metabolism is the following: as Ala is the most abundant amino acid in hornet silk (up to $30 \%$ of the amino-acid composition), ${ }^{10}$ a large amount of Ala is consumed during the biosynthesis of hornet silk. Therefore, if the silk proteins were biosynthesized under low Ala uptake, transamination from Ala to other amino acids would likely be repressed. Actually, the larval saliva used as the labeling larvae bait in this study contains only $6 \% \mathrm{Ala}^{22}$

\section{Quantitative analysis of the fraction of Ala in an $\alpha$-helical} conformation in hornet silk

In Figure 3, the expanded $\mathrm{Ala} C=\mathrm{O}, \mathrm{C}_{\alpha}$ and $\mathrm{C}_{\beta}$ regions of the ${ }^{13} \mathrm{C}$ CP-MAS spectra are shown for unlabeled and labeled cocoons with thin and thick lines, respectively. In the Ala $C_{\alpha}$ peak region, the spectra of hornet cocoons labeled at two different labeling rates (two
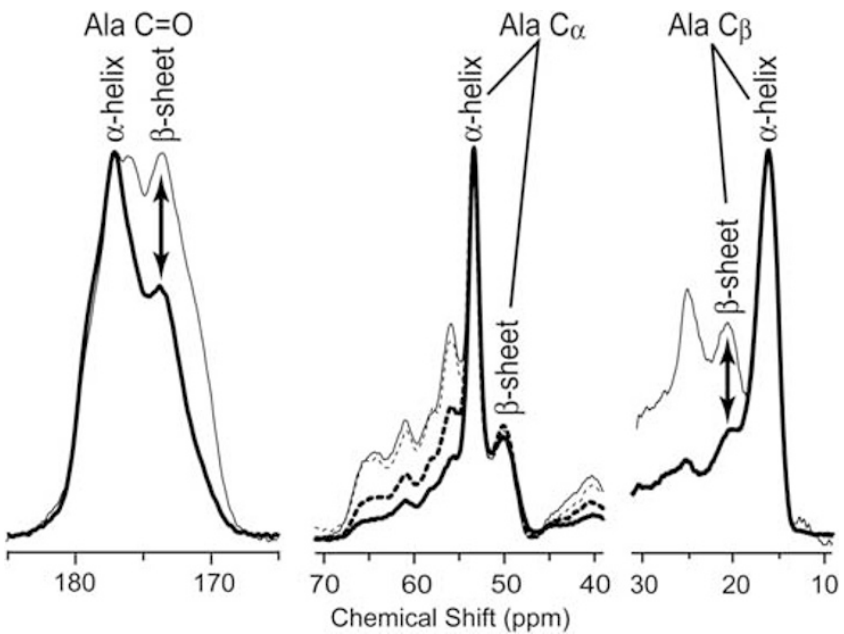

Figure 3 Expansion of the Ala $\mathrm{C}=0, \mathrm{C}_{\alpha}$ and $\mathrm{C}_{\beta}$ regions of the ${ }^{13} \mathrm{C} \mathrm{CP}$-MAS NMR spectra of hornet cocoons produced by larvae (solid thin lines) and $D L-A l a-{ }^{13} C_{3}$ administered larvae (solid thick lines) of $V$. mandarinia. The Ala $\mathrm{C}_{\alpha}$ region of the spectra of hornet cocoons produced by $V$. simillima xanthoptera Cameron larva (dotted thin line) and another $\mathrm{DL}-\mathrm{Ala}_{-}{ }^{1{ }^{3} \mathrm{C}_{3}}$ administered $V$. mandarinia larva with different labeling rate (dotted thick line) is also shown. All the peaks attributable to Ala $\mathrm{C}=0, \mathrm{C}_{\alpha}$ and $\mathrm{C}_{\beta}$ in an $\alpha$-helical structure are adjusted to the same intensity. Arrows indicate the difference in the peak intensities between the unlabeled and labeled cocoons. 
thick spectra of Ala $\mathrm{C}_{\alpha}$ peak regions in Figure 3) and two unlabeled cocoons from two different hornet species, $V$. mandarinia and $V$. simillima xanthoptera Cameron (two thin spectra of Ala $\mathrm{C}_{\alpha}$ peak region in Figure 3), are shown. In all of the spectra in Figure 3, all the peaks attributable to Ala $\mathrm{C}=\mathrm{O}, \mathrm{C}_{\alpha}$ and $\mathrm{C}_{\beta}$ in an $\alpha$-helical structure were adjusted to the same intensity.

In Figure 3, the difference between the unlabeled and labeled cocoons can be seen in the peak intensities of $\mathrm{Ala} C=\mathrm{O}$ and $\mathrm{C}_{\beta}$ in $\beta$-sheets (indicated by arrows). These differences were due to the overlap of Ala $\mathrm{C}=\mathrm{O}$ and $\mathrm{C}_{\beta}$ peaks with other amino-acid peaks. If no other peak overlapped with the $\mathrm{Ala} \mathrm{C}=\mathrm{O}$ and $\mathrm{C}_{\beta}$ peaks, the intensity ratio of the $\beta$-sheet peak to $\alpha$-helix peak was unchanged even if the hornet silk was labeled at various rates. Overlap of $\mathrm{C}=\mathrm{O}$ peaks for Ala with those for other amino acids can be easily recognized because the $\mathrm{C}=\mathrm{O}$ peaks for all other amino acids appear in a narrow range from 170 to 180 p.p.m. On the other hand, $A l a C_{\beta}$ peaks are considered to overlap with the peaks of side-chain carbons of other amino acids, such as Thr $\mathrm{C}_{\gamma}$ and Val $\mathrm{C}_{\gamma} \cdot{ }^{25,26}$

In contrast, in case of Ala $\mathrm{C}_{\alpha}$, the line shapes of the $\alpha$-helix and $\beta$-sheet peaks completely overlap in all of spectra (Figure 3 ), which indicates that the intensity ratio of the peaks in $\alpha$-helical and $\beta$-sheet structures was identical in both unlabeled and labeled cocoons and in cocoons labeled at different labeling rates. This correspondence was due to the fact that no other peak overlapped with the Ala $\mathrm{C}_{\alpha}$ peaks, which is significant for the analysis of the fraction of Ala in an $\alpha$-helical conformation in hornet silk. The abundance ratio of Ala residues in $\alpha$-helix and $\beta$-sheet can be estimated from the intensity ratio of the Ala $C_{\alpha}$ peaks in $\alpha$-helix and $\beta$-sheet, if only the quantitative high-resolution ${ }^{13} \mathrm{C}$ NMR spectrum of hornet silk is obtained.

When the ${ }^{13} \mathrm{C}$ MAS NMR spectrum was obtained using the DP method with a repetition time of over $5 \times \mathrm{T}_{1}(\mathrm{C})$, the intensity ratios of each peak corresponded exactly to the abundance ratio, where $T_{1}(C)$ is the longitudinal relaxation time of ${ }^{13} \mathrm{C}$. The $\mathrm{T}_{1}(\mathrm{C}) \mathrm{s}$ of the Ala $\mathrm{C}_{\alpha}$ in $\alpha$-helices and $\beta$-sheets, determined by Torchia NMR pulse sequence, ${ }^{27}$ had a value of $5 \mathrm{~s}$. Therefore, the ${ }^{13} \mathrm{C}$ DP-MAS spectrum for quantitative analysis of Ala $\mathrm{C}_{\alpha}$ peaks can be obtained when the repetition time sets over $25 \mathrm{~s}$. The peak intensities of $\mathrm{Ala}_{\alpha} \mathrm{C}_{\alpha}$ in the ${ }^{13} \mathrm{C}$ DP-MAS NMR spectrum with a repetition time of $25 \mathrm{~s}$ were deconvoluted into two peaks-one at 53.4 p.p.m., which was attributable to Ala in an $\alpha$-helical structure, and one at 50.2 p.p.m., which was attributable to $\beta$-sheet. From these deconvolution data, the fraction of Ala residues in $\alpha$-helices was estimated to be $56 \%$ (Figure 4 ).

In order to generalize the method used to determine the fraction of Ala residues in the $\alpha$-helices in hornet silk, the affect of the NMR measurement conditions on the Ala fraction value was examined. Although isotope-labeled cocoons were used in this study, when unlabeled cocoons are used as the sample, with a repetition time of $25 \mathrm{~s}$, it is difficult to obtain a sufficient signal-to-noise spectrum to conduct peak deconvolution in detail within the limited amount of time. As described above, the $T_{1}(C)$ s of the Ala $C_{\alpha}$ in $\alpha$-helices and $\beta$-sheets were identical, which indicates that the intensity ratio of the peaks of the Ala $C_{\alpha}$ in $\alpha$-helices and $\beta$-sheets in ${ }^{13} \mathrm{C}$ DP-MAS NMR spectrum is identical independent of the repetition time. Predictably, the intensity ratio of the peaks of the Ala $\mathrm{C}_{\alpha}$ in $\alpha$-helices and $\beta$-sheets in ${ }^{13} \mathrm{C}$ DP-MAS NMR spectra at various repetition times were almost identical (Figure 4). This result indicates that the ${ }^{13} \mathrm{C}$ DP-MAS spectrum for quantitative analysis of Ala $\mathrm{C}_{\alpha}$ peaks can be obtained, even with a shorter repetition time.

On the other hand, the CP method has an advantage for obtaining ${ }^{13} \mathrm{C}$ NMR spectrum under shorter repetition times. However, the

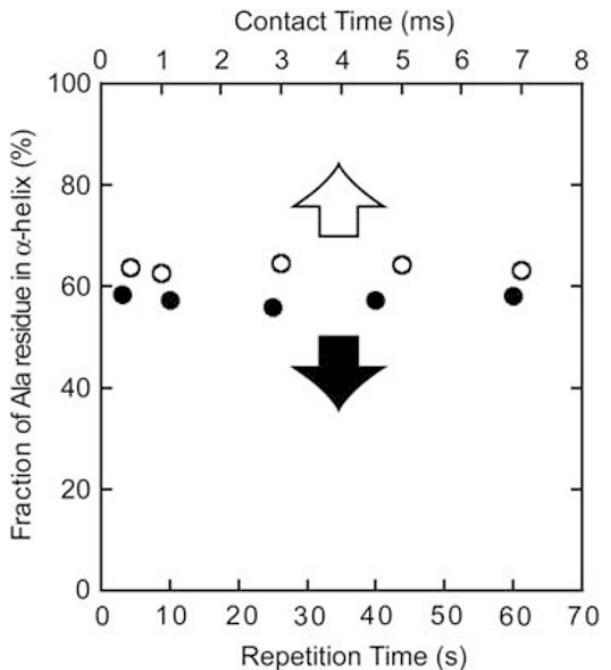

Figure 4 The fractions of Ala resides in $\alpha$-helices determined from the ${ }^{13} \mathrm{C}$ DP-MAS NMR spectra of hornet silk as a function of repetition time (0) and from the ${ }^{13} \mathrm{C}$ CP-MAS NMR spectra as a function of contact time $(O)$.

peaks obtained using the CP method do not necessarily always exactly correspond to the abundance ratio. ${ }^{28,29}$ Thus, the values for the Ala fraction in an $\alpha$-helical conformation determined from the peak intensities of the Ala $\mathrm{C}_{\alpha}$ in ${ }^{13} \mathrm{C}$ CP-MAS NMR spectra at various contact times were compared with those determined from ${ }^{13} \mathrm{C}$ DPMAS NMR spectra (Figure 4). It was found that the Ala fraction values are independent of contact time and almost identical to those determined using the DP method. This result indicates that a quantitative analysis of the Ala $\mathrm{C}_{\alpha}$ peaks in hornet silk can be performed using the ${ }^{13} \mathrm{C}$ CP-MAS NMR spectra at any contact time. On the basis of the above-mentioned results, the fraction of Ala in an $\alpha$-helical conformation in hornet silk was estimated to be approximately $60 \%$, a value that is relatively consistent independent of the NMR method and conditions used.

Furthermore, because the line shape of the spectrum in the Ala $\mathrm{C}_{\alpha}$ region of the $V$. mandarinia cocoon was in complete accordance with that for the V. simillima xanthoptera Cameron cocoon (Figure 3), it can be said that the fraction of $\alpha$-helical Ala in these species is identical. It is known that the hornet silks produced by these two species have similar protein compositions. ${ }^{9}$ Consequently, we confirmed that the fraction of Ala in $\alpha$-helices in the hornet silks produced by $V$. mandarinia and $V$. simillima xanthoptera Cameron larvae is also approximately $60 \%$.

\section{Molecular structure of hornet silk proteins}

Ala is the most abundant amino acid in hornet silk. Although Ala residues are most abundant in the Ala-rich domain, they are also found to a lesser extent in the Ser-rich domains. Figure 5 shows the fraction of Ala residues in coiled-coil domains, Ala-rich domains and Ser-rich domains in the four major silk proteins (Vssilk 1-4) of $V$. simillima xanthoptera Cameron, which was determined by inputting their amino-acid sequences into PROSITE and MARCOIL programs. This figure shows that close to $20 \%$ of the Ala residues in Vssilk 1-4 exists in Ser-rich domains, suggesting that the structural information obtained from the Ala peaks in the NMR spectra of hornet silk includes structural information not only for the Ala-rich domains but also for the Ser-rich domains. 


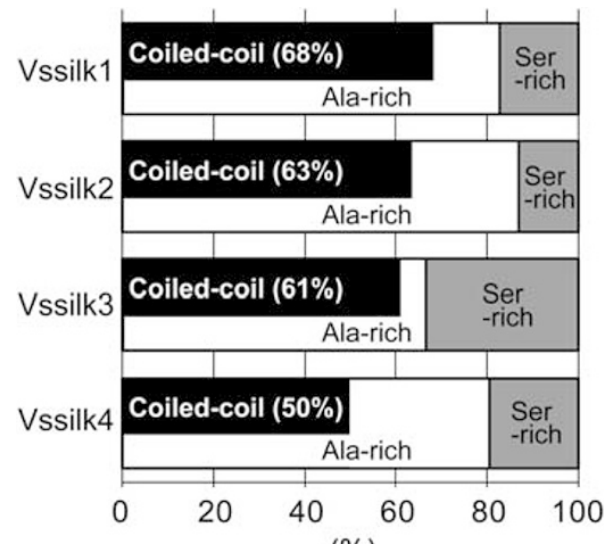

(\%)

Figure 5 Fractions of Ala residues within coiled-coil domains in the Ala-rich and Ser-rich domains of the four major proteins (Vssilk 1-4) of $V$. simillima xanthoptera Cameron were determined from their amino-acid sequences by MARCOIL and PROSITE programs.

Figure 5 shows that approximately $60 \%$ of the Ala residues in Vssilk 1-4 exist in coiled-coil domains. A prerequisite for the formation of a coiled-coil structure is that protein chains form an $\alpha$-helix conformation. Therefore, if $60 \%$ of Ala residues are within a coiled-coil structure, the fraction of Ala residues in an $\alpha$-helical conformation must be equal to or greater than $60 \%$. It should be noted that this coiled-coil Ala fraction $(60 \%)$ is identical to the fraction of Ala in $\alpha$-helices determined by solid-state NMR (Figure 4 ). Based on this finding, most of the Ala residues in $\alpha$-helices are found in the coiled-coil region; that is, the amount of $\alpha$-helical Ala without or outside coiled-coil formations is small. As the coiled-coil structure only exists in the Ala-rich domain and not in the Ser-rich domain (Figure 5), $\alpha$-helices mostly form in the Ala-rich domain, and infrequently form in the Ser-rich domains. We deduce that the Serrich domains in hornet silk would preferentially adopt a $\beta$-sheet conformation.

Coiled-coil prediction analysis indicated that the potential coiledcoil domains are located only in the central portion of the protein chains of the major hornet silk proteins. Protein domain prediction analysis indicated that Ala-rich domains are the central portion of protein chains of the major hornet silk proteins, whereas Ser-rich domains are the ends of the protein chains. Therefore, we deduce that the $\alpha$-helical conformation mostly forms in the central portion of the hornet silk chains, whereas the ends of the protein chains would preferentially adopt a $\beta$-sheet conformation.

\section{CONCLUSION}

The conclusions of the present study are summarized below:

(1) Although the hornet is a social insect and all larval baits are brought by adult hornets, we could breed hornet fifth-instar larvae until the larvae spin hornet silk, by artificially feeding larvae saliva. By feeding the larvae saliva mixed with isotopelabeled $D L-\mathrm{Ala}^{13} \mathrm{C}_{3},\left[{ }^{13} \mathrm{C}_{3}\right]$ Ala was incorporated into the hornet silk, and $\left[{ }^{13} \mathrm{C}_{3}\right] \mathrm{Ala}$-enriched hornet silk was obtained.

(2) The enriched hornet silk is suitable for investigation via NMR. We proposed that the Ala $\mathrm{C}_{\alpha}$ peaks of the CP- and DP-MAS NMR spectra of hornet silk could be used to determine the quantitative ratio between Ala residues in $\alpha$-helices and $\beta$-sheets.
(3) From the quantitative NMR analysis of the Ala $\mathrm{C}_{\alpha}$ peaks and the bioinformatics analysis of hornet silk, Ala residues in an $\alpha$-helical conformation were found to exist primarily in the center of the protein chains, the Ala-rich domains, of hornet silk. In contrast, both ends of the protein chains, the Ser-rich domains, are nearly devoid of $\alpha$-helical structure. Therefore, we confirmed that the Ser-rich domains in hornet silk preferentially adopt a $\beta$-sheet conformation. The finding that the Ser-rich domain in hornet silk preferentially adopts a $\beta$-sheet conformation gives an important clue to elucidate the mechanisms underlying hornet silk formation during larval spinning, deformation when drawing the hornet silk materials and hornet silk gel formation in aqueous solution.

\section{ACKNOWLEDGEMENTS}

This work was supported in part by the Japan Society for the Promotion of Science (Grant No. 21580072), and the Agri-Health Translational Research Project and Research and Development Project for applications promoting new polices for Agriculture Forestry and Fisheries.

1 Craig, C. L. \& Riekel, C. Comparative architecture of silks, fibrous proteins and their encoding genes in insects and spiders. Comp. Biochem. Physiol. B Biochem. Mol. Biol. 133, 493-507 (2002)

2 Rudall, K. M. \& Kenchington, W. Arthropod silks: the problem of fibrous proteins in animal tissues. Annu. Rev. Entomol 16, 73-96 (1971).

3 Sutherland, T. D., Young, J. H., Weisman, S., Hayashi, C. Y. \& Merritt, D. J. Insect silk: one name, many materials. Annu. Rev. Entomol. 55, 171-188 (2010).

4 Kirshboim, S. \& Ishay, J. S. Silk produced by hornets: thermophotovoltaic properties - a review. Comp. Biochem. Physiol. A Mol. Integr. Physiol. 127, 1-20 (2000).

5 Kameda, T., Kojima, K., Zhang, Q. \& Sezutsu, H. Identification of hornet silk gene with a characteristic repetitive sequence in Vespa simillima xanthoptera. Comp. Biochem. Physiol. B Biochem. Mol. Biol. 161, 17-24 (2012).

6 Kameda, T., Kojima, K., Togawa, E., Sezutsu, H., Zhang, Q., Teramoto, H. \& Tamada, Y Drawing-induced changes in morphology and mechanical properties of hornet silk gel films. Biomacromolecules 11, 1009-1018 (2010).

7 Kameda, T., Kojima, K., Sezutsu, H., Zhang, Q., Teramoto, H. \& Tamada, Y. Hornet (Vespa) silk composed of coiled-coil proteins. Kobunshi Ronbunshu 67, 641-635 (2010).

8 Kameda, T. \& Tamada, Y Variable-temperature ${ }^{13} \mathrm{C}$ solid-state NMR study of the molecular structure of honeybee wax and silk. Int. J. Biol. Macromol. 44, 64-69 (2009).

9 Kameda, T., Kojima, K., Zhang, Q., Sezutsu, H., Teramoto, H., Kuwana, Y. \& Tamada, Y. Hornet silk proteins in the cocoons produced by different Vespa species inhabiting Japan. Comp. Biochem. Physiol. B Biochem. Mol. Biol. 151, 221-224 (2008).

10 Sezutsu, H., Kajiwara, H., Kojima, K., Mita, K., Tamura, T., Tamada, Y. \& Kameda, T. Identification of four major hornet silk genes with a complex of alanine-rich and serinerich sequences in Vespa simillima xanthoptera Cameron. Biosci. Biotechnol. Biochem. 71, 2725-2734 (2007).

11 Kameda, T., Niizeki, T. \& Sonoyama, M. In-situ observation of tryptophan in larval cocoon silk of the hornet Vespa simillima xanthoptera cameron by ultraviolet resonance raman spectroscopy. Biosci. Biotechnol. Biochem. 71, 1353-1355 (2007).

12 Kameda, T., Kojima, K., Miyazawa, M. \& Fujiwara, S. Film formation and structural characterization of silk of the hornet Vespa simillima xanthoptera Cameron. Zeitschrift fur Naturforschung. Sect. C. J. Biosci. 60, 906-914 (2005)

13 Sutherland, T. D., Church, J. S., Hu, X. A., Huson, M. G., Kaplan, D. L. \& Weisman, S Single honeybee silk protein mimics properties of multi-protein silk. Plos One 6 , e16489 (2011)

14 Crick, F. H. C. The packing of alpha-helices - simple coiled-coils. Acta Crystallographica 6, 689-697 (1953).

15 Cohen, C. \& Parry, D. A. D. Alpha-helical coiled coils and bundles - how to design an alpha-helical protein. Proteins Struct. Funct. Genet. 7, 1-15 (1990).

16 Spek, E. J., Olson, C. A., Shi, Z. S. \& Kallenbach, N. R. Alanine is an intrinsic alphahelix stabilizing amino acid. J. Am. Chem. Soc. 121, 5571-5572 (1999).

17 Fujiwara, T., Kobayashi, Y., Kyogoku, Y. \& Kataoka, K. Conformational study of ${ }^{13} \mathrm{C}$ enriched fibroin in the soild-state, using the cross polarization nuclear-magneticresonance method. J. Mol. Biol. 187, 137-140 (1986).

18 Asakura, T., Demura, M., Nagashima, M., Sakaguchi, R., Osanai, M. \& Ogawa, K. Metabolic flux and incorporation of $2-{ }^{13} \mathrm{C}$ glycine into silk fibroin studied by ${ }^{13} \mathrm{C}$ NMR invivo and invitro. Insect Biochem. 21, 743-748 (1991).

19 Osanai, M., Okudaira, M., Naito, J., Demura, M. \& Asakura, T. Biosynthesis of $\mathrm{L}$-alanine, a major amino acid of fibroin in Samia cynthia ricini. Insect Biochem. Mol. Biol. 30, 225-232 (2000) 
20 Creager, M. S., Izdebski, T., Brooks, A. E. \& Lewis, R. V. Elucidating metabolic pathways for amino acid incorporation into dragline spider silk using ${ }^{13} \mathrm{C}$ enrichment and solid state NMR. Comp. Biochem. Physiol. A Mol. Integr. Physiol. 159, 219-224 (2011).

21 Hess, S., van Beek, J. \& Pannell, L. K. Acid hydrolysis of silk fibroins and determination of the enrichment of isotopically labeled amino acids using precolumn derivatization and high-performance liquid chromatography-electrospray ionizationmass spectrometry. Anal. Biochem. 311, 19-26 (2002).

22 Abe, T., Takiguchi, Y., Tamura, M., Shimura, J. \& Yamazaki, K. Effects of Vespa amino acid mixture (VAAM) isolated from hornet larval saliva and modified VAAM nutrients on endurance exercise in swimming mice - improvement in performance and changes of blood lactate and glucose —. Japanese J. Phys. Fitness Sports Med. 44, 225-238 (1995).

23 Abe, T., Tanaka, Y., Miyazaki, H. \& Kawasaki, Y. Y. Comparative-study of the composition of hornet larval saliva, its effect on behavior and role of Trophallaxis. Comp. Biochem. Physiol. C Pharmacol. Toxicol. Endocrinol. 99, 79-84 (1991).
24 Voet, D. \& Voet, J. G. Biochemistry. 3rd Edition (Wiley \& Sons, Hoboken, NJ, 2004).

25 Teramoto, H., Kakazu, A. \& Asakura, T. Native structure and degradation pattern of silk sericin studied by C-13 NMR spectroscopy. Macromolecules 39, 6-8 (2006).

26 Kricheldorf, H. R. \& Muller, D. Secondary structure of peptides $16^{\text {th }}$. Characterization of proteins by means of ${ }^{13} \mathrm{C}$ NMR CP/MAS spectroscopy. Colloid Polym. Sci 262, 856-861 (1984).

27 Torchia, D. A. Measurement of proton-enhanced ${ }^{13} \mathrm{C} \mathrm{T}_{1}$ values by a method which suppresses artifacts. J. Magnet. Resonance 30, 613-616 (1978).

28 Kameda, T. Molecular structure of crude beeswax studied by solid-state ${ }^{13} \mathrm{C}$ NMR. J. Insect Sci. 4, 29 (2004).

29 Kameda, T., Miyazawa, M. \& Murase, S. Conformation of drawn poly(trimethylene terephthalate) studied by solid-state ${ }^{13} \mathrm{C}$ NMR. Magnetic Resonance Chem. 43, 21-26 (2005). 